\title{
Thermodynamics of polynuclear aromatic molecules \\ I. Heat capacities and enthalpies of fusion of pyrene, fluoranthene, and triphenylene ${ }^{\dagger}$
}

\author{
WEN-KUEI WONG $\ddagger$ and EDGAR F. WESTRUM, Jr.\& \\ Department of Chemistry, University of Michigan, \\ Ann Arbor, Michigan 48104, U.S.A.
}

(Received 14 August 1970)

\begin{abstract}
The heat capacities of three crystalline condensed-ring aromatic substances were studied from 5 to $350 \mathrm{~K}$ by adiabatic cryogenic calorimetry and into the liquid phase with the intermediate-range adiabatic calorimeter. The heat capacities, entropies, and enthalpies at $298 \mathrm{~K}$, together with the triple points and enthalpies of melting are:

\begin{tabular}{|c|c|c|c|c|c|}
\hline & $\frac{C_{\mathrm{s}}}{\mathrm{cal} \mathrm{mol} \mathrm{mol}^{-1} \mathrm{~K}^{-1}}$ & $\frac{S^{\circ}}{\text { cal } \mathrm{mol}^{-1} \mathrm{~K}^{-1}}$ & $\frac{H^{\circ}-H_{0}^{\circ}}{\text { cal mol-1 }}$ & $\frac{T_{\mathrm{m}}}{\mathrm{K}}$ & $\frac{\Delta H_{\mathrm{m}}}{\mathrm{cal} \mathrm{\textrm {mol } ^ { - 1 }}}$ \\
\hline & 54.90 & 53.75 & 8040 & 423.81 & 4148 \\
\hline & 55.03 & 55.11 & 8191 & 383.34 & 4477 \\
\hline enylene & 61.95 & 60.87 & 9205 & & \\
\hline
\end{tabular}
\end{abstract}

A gradual transition in pyrene occurs near $120.8 \mathrm{~K}$ with an associated enthalpy increment of $68.8 \mathrm{cal} \mathrm{mol}^{-1}$. The nature of the melting process is considered.

\section{Introduction}

It is generally appreciated that the physicochemical properties of the condensed polynuclear aromatic compounds are closely correlated with the number of either carbon atoms or $\pi$-electrons. The linear dependence of electrical conductivity, ${ }^{(1)}$ the enthalpy of sublimation, ${ }^{(2)}$ as well as the logarithmic dependence of the reciprocal density ${ }^{(3)}$ from benzene through circumanthracene $\left(\mathrm{C}_{40} \mathrm{H}_{16}\right)$, correlate closely with the number of carbon atoms. The densities relate to that of graphite, which may be considered to be the upper bound for this type of molecule. There is also a progressive shift in color with increasing complexity of the molecule. Although some thermochemical data on these substances are reported in the literature, thermal data are not available for compounds of greater complexity than pyrene. Since the heatcapacity determination provides a powerful tool for the study of phase transitions,

† This work was supported in part by the Division of Research, United States Atomic Energy Commission.

‡ Abstracted from a dissertation submitted to the Horace H. Rackham School of Graduate Studies at the University of Michigan in partial fulfillment of the requirements for the Ph.D. degree.

$\S$ To whom correspondence concerning this work should be addressed. 
as well as the basic data for chemical thermodynamic functions useful in science and technology, the present study is the first in a series of investigations intended to provide insight into the thermal properties of these interesting molecules. Rather low-precision measurements of the heat capacity of pyrene have been reported ${ }^{(4)}$ over the region 90 to $300 \mathrm{~K}$. The present investigation reports both low- and intermediate-temperature heat capacities extending through fusion into the liquid phase for pyrene $\left(\mathrm{C}_{16} \mathrm{H}_{10}\right)$ fluoranthene $\left(\mathrm{C}_{16} \mathrm{H}_{10}\right)$, and triphenylene $\left(\mathrm{C}_{18} \mathrm{H}_{12}\right)$ whose structures are shown below.

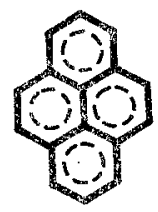

Pyrene

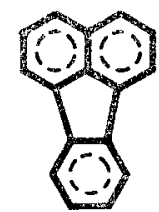

Fluoranthene

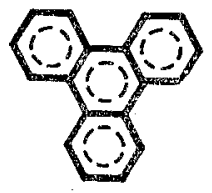

Triphenylene

\section{Experimental}

\section{PURIFICATION AND CHARACTERIZATION OF THE SAMPLES}

Pyrene. Crude, orange-colored material, labelled 98 per cent pure and obtained from Rütgerswerk-Aktiegesellschaft, was fractionally recrystallized eight times from p-dioxane. The pale yellow crystals obtained thereby were dried under vacuum at $300 \mathrm{~K}$ for several days and fractionally resublimed trap-to-trap in vacuum six times from about 400 to $200 \mathrm{~K}$, with the more volatile impurities removed by a liquid nitrogen trap. The massive crystalline sublimate was shattered into colorless fragments by quenching the Pyrex receiver in liquid nitrogen. Microchemical analyses on the resulting material indicated 95.00 mass per cent of $\mathrm{C}$ and 4.99 mass per cent of $\mathrm{H}$ (theoretical: 95.02 mass per cent of $\mathrm{C}$ and 4.98 mass per cent of $\mathrm{H}$ ). The mole fraction of the liquid-soluble, solid-insoluble impurity determined by fractional fusion was 0.0003 .

Fluoranthene. Fluoranthene, obtained from the Aldrich Chemical Co., Inc., was subjected to five fractional recrystallizations from benzene and four successive gradient sublimations in vacuum. Preliminary measurements were made in the intermediate-temperature calorimeter through fusion at this stage of purification (0.0039 mole fraction impurity) and are designated "Impure Sample I" in subsequent tables. Finally, 15 passes of zone-refining were performed. The final product (Pure Sample II) had a faint yellowish tinge. Microchemical analyses indicated 95.02 mass per cent of $\mathrm{C}$ and 4.96 mass per cent of $H$ (theoretical: 95.02 mass per cent of $C$ and 4.98 mass per cent of $\mathrm{H}$ ). Fractional melting indicated a mole fraction of impurity of 0.00064 . Melting temperatures are discussed in the results section.

Triphenylene. A gray-colored, reputedly 99 moles per cent pure sample from K. \& K. Laboratories, Inc. was fractionally recrystallized five times from benzene and subjected to four successive gradient sublimations in vacuum. A total of 15 passes of 
zone-refining yielded a transparent crystal. Microchemical analyses indicated 94.74 mass per cent of $C$ and 5.28 mass per cent of $H$ (theoretical: 94.70 mass per cent of $C$ and 5.30 mass per cent of $\mathbf{H}$ ). Fractional fusion studies indicated a mole fraction of impurity of 0.00038 and a triple point temperature for the sample of $471.01 \mathrm{~K}$.

\section{CALORIMETRIC APPARATUS}

The cryostats, calorimeters, and designations of the capsule-type platinum-resistance thermometers, together with the sample masses, pressures of helium gas added to facilitate thermal conduction, and the percentages of total heat capacity due to sample, have been summarized in table 1 . The Mark II cryostat and the adiabatic technique

TABLE 1. Identification of calorimetric apparatus and quantities; $m$ denotes mass, $p$ pressure of added $\mathrm{He}, C_{\mathrm{s}}$ heat capacity, $M$ molar mass, and $\rho$ density.

\begin{tabular}{|c|c|c|c|c|c|c|}
\hline $\begin{array}{l}\text { Compound and } \\
\text { temperature range }\end{array}$ & $\begin{array}{l}\text { Cryostat } \\
\text { Mark }\end{array}$ & $\begin{array}{l}\text { Calori- } \\
\text { meter }\end{array}$ & $\begin{array}{l}\text { Thermo- } \\
\text { meter }\end{array}$ & $\frac{m \text { (sample) }}{\mathrm{g}}$ & $\frac{p(\mathrm{He})}{\text { Torr }}$ & $100 \frac{C_{\mathrm{s}}(\text { sample })}{C_{\mathrm{s}}(\text { total })}$ \\
\hline $\begin{array}{l}\text { Pyrene } \\
M=202.2581 \mathrm{~g} \mathrm{~mol}^{-} \\
\rho=1.27 \mathrm{~g} \mathrm{~cm}^{-3}(11) \\
\\
4.7 \text { to } 318 \mathrm{~K} \\
\\
279 \text { to } 484 \mathrm{~K}\end{array}$ & $\begin{array}{l}\text { II } \\
\text { IV }\end{array}$ & $\begin{array}{l}W-28 \\
W-22\end{array}$ & $\begin{array}{l}\text { A-5 } \\
\text { A-7 }\end{array}$ & $\begin{array}{l}55.312 \\
53.664\end{array}$ & $\begin{array}{l}111 \\
130\end{array}$ & $\begin{array}{l}94 \text { to } 54 \\
63 \text { to } 72\end{array}$ \\
\hline $\begin{array}{c}\text { Fluoranthene } \\
M=202.2581 \mathrm{~g} \mathrm{~mol}^{-} \\
p=1.243 \mathrm{~g} \mathrm{~cm}^{-3}(12) \\
\text { Impure Sample (I) } \\
297 \text { to } 457 \mathrm{~K}\end{array}$ & IV & W-22 & A-7 & 91.274 & 99 & \\
\hline $\begin{array}{c}\text { Pure Sample (II) } \\
4.6 \text { to } 348 \mathrm{~K} \\
291 \text { to } 427 \mathrm{~K}\end{array}$ & $\begin{array}{l}\text { II } \\
\text { IV }\end{array}$ & $\begin{array}{l}W-28 \\
W-22\end{array}$ & $\begin{array}{l}A=5 \\
A-7\end{array}$ & $\begin{array}{l}63.563 \\
79.512\end{array}$ & $\begin{array}{l}133 \\
146\end{array}$ & $\begin{array}{c}95 \text { to } 60 \\
74\end{array}$ \\
\hline $\begin{array}{l}\text { Triphenylene } \\
\begin{aligned} M= & 228.2963 \mathrm{~g} \mathrm{~mol}^{-} \\
\rho= & 1.30 \mathrm{~g} \mathrm{~cm}^{-3}(13) \\
& 4.7 \text { to } 339 \mathrm{~K} \\
& 298 \text { to } 514 \mathrm{~K}\end{aligned}\end{array}$ & II & $\begin{array}{l}W-28 \\
W-22\end{array}$ & $\begin{array}{l}\text { A-5 } \\
\text { A-8 }\end{array}$ & $\begin{array}{l}66.526 \\
66.798\end{array}$ & $\begin{array}{r}145 \\
95\end{array}$ & $\begin{array}{l}94 \text { to } 60 \\
70 \text { to } 79\end{array}$ \\
\hline
\end{tabular}

employed for cryogenic calorimetry (4 to $350 \mathrm{~K}$ ) have been described, ${ }^{(5)}$ as have the intermediate-temperature Mark IV thermostat and the adjuvant techniques. ${ }^{(6)}$ Calorimeter W-28 has a soldered closure similar to that described elsewhere; ${ }^{(7)}$ calorimeter W-22, provided with a gold-gasketed seal, has been detailed. ${ }^{(6)}$ To avoid exposure of the sample to air, the calorimeters were loaded in a nitrogen-atmosphere anhydrous chamber. Each calorimeter was then connected to a high-vacuum line, highly evacuated, degassed and sealed either by soldering or by remote mechanical closure of the gasketed screw-cap after adding the conduction helium to enhance thermal contact between calorimeter and sample. Where necessary in the cryogenic calorimetry, the amounts of Apiczon-T grease, Cerroseal solder, and helium gas were adjusted to correspond to the quantities present during the separate heat-capacity determinations on the empty calorimeter + heater + thermometer assembly. 
Automatic adiabatic shield control is used in both instruments and consists of three separate channels of recording circuitry provided with proportional, rate, and reset actions which maintain the null thermal head between calorimeter and shield within $0.001 \mathrm{~K}$.

Accuracy is assured by ultimately referring all determinations of mass, temperature, resistance, and potential to calibrations and standardizations performed by the National Bureau of Standards and to test measurements on heat-capacity standards established by the Calorimetry Conference.

\section{Results}

\section{HEAT CAPACITIES}

The experimental values of the molar heat capacities of these three substances are summarized in table 2 and presented graphically in figure 1. All data in this and subsequent tables are derived in terms of the defined thermochemical calorie equal to $4.184 \mathrm{~J}$ and an ice point of $273.15 \mathrm{~K}$. The heat capacities are listed in chronological

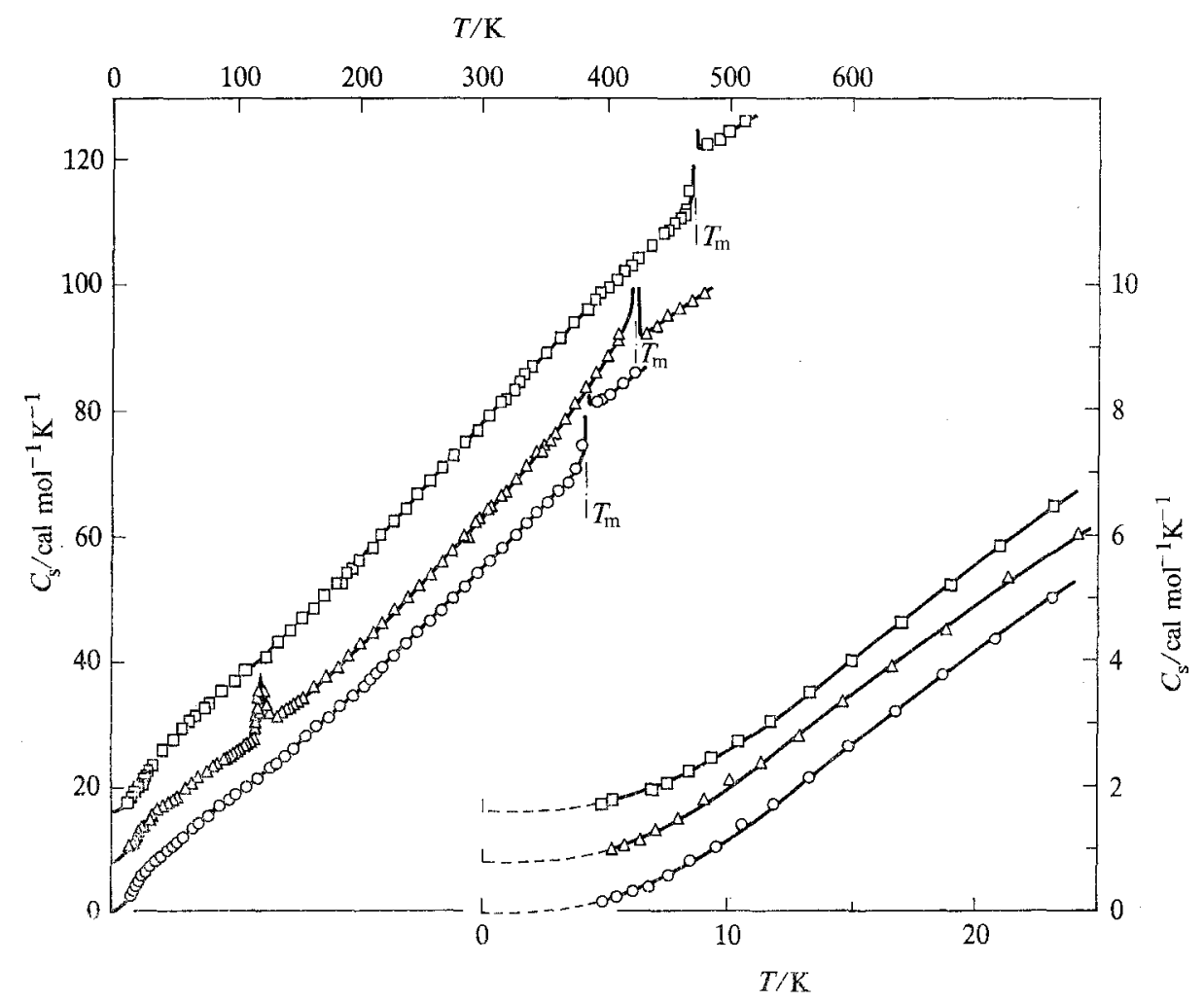

FIGURE 1. The heat capacities of pyrene $\triangle$, fluoranthene $\bigcirc$, and triphenylene $\square$. The ordinates for each curve other than that of fluoranthene are successively displaced by $8 \mathrm{cal} \mathrm{mol}^{-1} \mathrm{~K}^{-1}$ for each curve on the left and by $0.8 \mathrm{cal} \mathrm{mol}{ }^{-1} \mathrm{~K}^{-1}$ for the enlarged-scale curves on the right. The points indicated represent the experimental determinations. 
TABLE 2. Heat capacities of pyrene, fluoranthene, and triphenylene

\begin{tabular}{|c|c|c|c|c|c|c|c|}
\hline$\frac{\langle T\rangle}{\mathbf{K}}$ & $\frac{C_{\mathrm{s}}}{\mathrm{calmol} \mathrm{mol}^{-1} \mathrm{~K}^{-1}}$ & $\frac{\langle T\rangle}{\overline{\mathrm{K}}}$ & $\frac{C_{\mathrm{s}}}{\text { cal mol }{ }^{-1} \mathrm{~K}^{-1}}$ & $\frac{\langle T\rangle}{\mathbf{K}}$ & $\frac{C_{\mathrm{s}}}{\mathrm{cal} \mathrm{mol}^{-1} \mathrm{~K}^{-1}}$ & $\frac{\langle T\rangle}{\mathrm{K}}$ & $\frac{C_{\mathrm{s}}}{\mathrm{cal} \mathrm{mol}^{-1} \mathrm{~K}^{-1}}$ \\
\hline \multicolumn{8}{|c|}{ Pyrene $\left(\mathrm{C}_{16} \mathrm{H}_{10}\right)$} \\
\hline \multicolumn{8}{|c|}{ Results using Mark II cryostat } \\
\hline \multicolumn{2}{|r|}{ Series I } & \multicolumn{2}{|c|}{ Series III } & 115.39 & 20.48 & 122.81 & 23.49 \\
\hline 5.27 & 0.173 & 93.88 & 17.06 & 116.16 & 20.91 & 124.82 & 34.35 \\
\hline 5.78 & 0.235 & 100.93 & 18.06 & 116.77 & 21.22 & 127.99 & 29.05 \\
\hline 6.40 & 0.319 & 107.60 & 19.07 & 117.37 & 21.68 & & \\
\hline 7.08 & 0.454 & 112.11 & 19.78 & 117.97 & 22.15 & \multicolumn{2}{|c|}{ Series VIII } \\
\hline 7.99 & 0.646 & 114.60 & 20.18 & 118.56 & 21.98 & \multirow{2}{*}{\multicolumn{2}{|c|}{$\Delta H_{\mathrm{t}}$ Run A }} \\
\hline 9.01 & 0.939 & 117.03 & 21.30 & 119.15 & 21.60 & & \\
\hline 10.10 & 1.254 & 119.14 & 32.56 & 119.73 & 23.97 & 119.41 & $\begin{array}{l}25.90 \\
31.44\end{array}$ \\
\hline 11.37 & 1.548 & 121.01 & 30.80 & 120.33 & 21.44 & 121.60 & 31.44 \\
\hline 12.90 & 1.987 & 123.08 & 23.29 & 120.92 & 22.62 & 123.73 & 28.16 \\
\hline 14.63 & 2.512 & 126.81 & 25.72 & 121.50 & 23.98 & 126.24 & 27.47 \\
\hline 16.60 & 3.089 & 132.63 & 23.72 & 122.08 & 21.85 & 129.53 & 23.92 \\
\hline 18.83 & 3.727 & 139.27 & 24.19 & 122.70 & 21.44 & 133.69 & 23.60 \\
\hline 21.30 & 4.411 & 146,02 & 25.28 & 123.29 & 21.52 & 138.63 & 24.09 \\
\hline 24.25 & 5.192 & 152.94 & 26.39 & 124.28 & 23.80 & & \\
\hline 27.97 & 6.111 & \multirow{2}{*}{\multicolumn{2}{|c|}{ Series IV }} & & & \multicolumn{2}{|c|}{ Series IX } \\
\hline 31.73 & 6.978 & & & \multirow{2}{*}{\multicolumn{2}{|c|}{ Series VI }} & 116.35 & 19.85 \\
\hline 35.32 & 7.761 & 151.35 & 26.13 & & & 117.69 & 20.71 \\
\hline 39.66 & 8.625 & 161.37 & 27.89 & 107.63 & 19.11 & 118.74 & 20.85 \\
\hline 44.21 & 9.468 & 171.04 & 29.62 & 114.45 & 20.22 & 119.78 & 20.95 \\
\hline 48.29 & 10.17 & 180.32 & 31.29 & 116.00 & 20.69 & 120.82 & 21.20 \\
\hline 52.87 & 10.93 & 189.46 & 32.97 & 117.50 & 22.35 & 121.85 & 21.32 \\
\hline & & 198.95 & 34.73 & 118.92 & 25.35 & 122.87 & 21.49 \\
\hline \multirow{2}{*}{\multicolumn{2}{|c|}{ Series II }} & 208.59 & 36.58 & 119.90 & 21.91 & 123.89 & 21.61 \\
\hline & & 218.05 & 38.42 & 120.86 & 27.08 & 124.90 & 21.82 \\
\hline 33.06 & 7.268 & 227.49 & 40.26 & \multirow{3}{*}{\multicolumn{2}{|c|}{ Series VII }} & 125.90 & 22.08 \\
\hline 38.54 & 8.401 & 236.75 & 42.16 & & & 126.90 & 22.14 \\
\hline 44.06 & 9.453 & 245.93 & 44.01 & & & 127.89 & 22.39 \\
\hline 48.80 & 10.27 & 255.23 & 45.86 & 82.07 & 15.36 & 128.87 & 22.49 \\
\hline 52.69 & 10.90 & 264.63 & 47.82 & 90.44 & 16.62 & $\begin{array}{l}120.07 \\
129.85\end{array}$ & 22.61 \\
\hline 56.72 & 11.54 & 274.08 & 49.82 & 99.27 & 17.85 & $\begin{array}{l}129.02 \\
131.50\end{array}$ & 22.85 \\
\hline 62.17 & 12.42 & 283.66 & 51.79 & 107.20 & 19.02 & 134.19 & 23.36 \\
\hline 68.10 & 13.30 & 293.63 & 54.02 & 113.84 & 20.11 & 138.43 & 24.02 \\
\hline 74.37 & 14.18 & 303.72 & 56.16 & 117.81 & 21.40 & $\begin{array}{l}150.43 \\
143.93\end{array}$ & $\begin{array}{l}24.02 \\
24.96\end{array}$ \\
\hline 81.74 & 15.30 & 313.50 & 58.36 & 118.83 & 20.89 & $\begin{array}{l}143.93 \\
150.04\end{array}$ & $\begin{array}{l}24.90 \\
25.93\end{array}$ \\
\hline 89.63 & 16.49 & & & 119.42 & 21.24 & 150.04 & 25.93 \\
\hline 93.88 & 17.06 & \multicolumn{2}{|c|}{ Series V } & 120.00 & 21.15 & \multirow{2}{*}{\multicolumn{2}{|c|}{ Series X }} \\
\hline 104.65 & 18.67 & 101.40 & 18.19 & 120.80 & 23.09 & & \\
\hline 111.55 & 19.70 & 114.15 & 20.24 & 121.81 & 23.90 & \multicolumn{2}{|c|}{$\Delta H_{\mathrm{t}}$ Run $\mathrm{B}$} \\
\hline \multicolumn{8}{|c|}{ Results using Mark IV thermostat } \\
\hline \multicolumn{2}{|c|}{ Series XI } & \multicolumn{2}{|c|}{ Series XII } & 334.91 & 63.10 & 391.35 & 77.64 \\
\hline 414.06 & 86.19 & 284.75 & 51.71 & 343.97 & 65.18 & 400.65 & 80.68 \\
\hline 421.15 & 178.8 & 294.70 & 54.11 & 353.21 & 67.18 & 409.82 & 83.97 \\
\hline 423.74 & 12700 & 304.34 & 56.32 & 362.26 & 69.75 & 416.61 & 87.84 \\
\hline 423.77 & 38600 & 313.74 & 58.55 & 371.28 & 72.15 & 421.65 & 168.5 \\
\hline 423.79 & 23400 & 316.98 & 59.07 & 380.50 & 74.70 & 423.62 & 4600 \\
\hline 426.31 & 180.5 & 326.05 & 61.22 & 389.78 & 77.43 & 423.72 & 15000 \\
\hline
\end{tabular}


TABLE 2-continued

\begin{tabular}{|c|c|c|c|c|c|c|c|}
\hline$\frac{\langle T\rangle}{\mathrm{K}}$ & 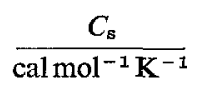 & $\frac{\langle T\rangle}{\mathbf{K}}$ & $\frac{C_{\mathrm{s}}}{\mathrm{cal} \mathrm{mol}^{-1} \mathrm{~K}^{-1}}$ & $\frac{\langle T\rangle}{\mathrm{K}}$ & $\frac{C_{\mathrm{s}}}{\text { cal } \mathrm{mol}^{-1} \mathrm{~K}^{-1}}$ & $\frac{\langle T\rangle}{\mathrm{K}}$ & $\frac{C_{\mathrm{s}}}{\mathrm{cal} \mathrm{mol}^{-1} \mathbf{K}^{-1}}$ \\
\hline \multicolumn{8}{|c|}{ Results using Mark IV Thermostat-continued } \\
\hline \multirow{5}{*}{$\begin{array}{l}423.75 \\
423.77 \\
423.78 \\
426.45\end{array}$} & \multirow{5}{*}{$\begin{array}{c}148000 \\
33500 \\
129000 \\
108.9\end{array}$} & 365.97 & 70.36 & 421.77 & 98.84 & 430.58 & 84.27 \\
\hline & & 374.92 & 72.87 & & & & \\
\hline & & & & \multicolumn{2}{|c|}{ Series XV } & \multicolumn{2}{|c|}{ Series XVII } \\
\hline & & \multirow{2}{*}{\multicolumn{2}{|c|}{ Series XIV }} & 409.32 & 83.95 & 430.70 & 84.21 \\
\hline & & & & $\Delta H_{\mathrm{m}} \mathrm{R}$ & $\operatorname{un} \mathrm{A}$ & 440.32 & 85.59 \\
\hline \multirow{2}{*}{\multicolumn{2}{|c|}{ Series XIII }} & 408.94 & 83.19 & \multirow{2}{*}{\multicolumn{2}{|c|}{ Series XVI }} & 449.86 & 86.89 \\
\hline & & 412.22 & 84.89 & & & 459.30 & 88.10 \\
\hline \multirow{2}{*}{$\begin{array}{l}347.37 \\
356.79\end{array}$} & 65.88 & 415.61 & 86.72 & 417.60 & 88.15 & 468.98 & 89.37 \\
\hline & 68.09 & 418.95 & 88.78 & $\Delta H_{\mathrm{m}} \mathrm{R}$ & an $B$ & 478.79 & 90.77 \\
\hline
\end{tabular}

Fluoranthene $\left(\mathrm{C}_{16} \mathrm{H}_{10}\right)$

Impure sample I-results using Mark IV calorimetric thermostat

\begin{tabular}{|c|c|c|c|c|c|c|c|}
\hline \multicolumn{2}{|c|}{ Series I } & \multicolumn{2}{|c|}{ Series II } & \multicolumn{2}{|c|}{ Series IV } & 423.29 & 85.28 \\
\hline 302.15 & 55.85 & 372.92 & 73.92 & 377.23 & 90.44 & 432.68 & 86.56 \\
\hline 311.57 & 57.64 & 379.92 & 170.8 & 381.71 & $464^{-17}$ & 442.15 & 87.76 \\
\hline 321.31 & 59.72 & 382.95 & 3950 & 382.73 & 2960 & 451.72 & 88.91 \\
\hline 331.20 & 61.66 & 383.05 & 10620 & 383.04 & 14100 & \multirow{2}{*}{\multicolumn{2}{|c|}{ Series VI }} \\
\hline 341.20 & 63.63 & 384.87 & 229.3 & 383.10 & 17400 & & \\
\hline 350.94 & 65.70 & 391.01 & 81.08 & 386.12 & 122.8 & $\Delta H$ Run & \\
\hline 360.44 & 67.80 & & & & & 373.88 & 74.80 \\
\hline 370.01 & 70.60 & \multirow{2}{*}{\multicolumn{2}{|c|}{ Series III }} & \multicolumn{2}{|c|}{ Series V } & $\Delta H_{f}$ Run & \\
\hline 378.43 & 105.7 & & & \multirow{2}{*}{\multicolumn{2}{|c|}{$\begin{array}{l}\Delta H \text { Run } \\
\Delta H_{\mathrm{f}} \text { Run }\end{array}$}} & \multirow{2}{*}{\multicolumn{2}{|c|}{ Series VII }} \\
\hline 382.44 & 831.0 & 365.09 & 68.95 & & & & \\
\hline & & 370.49 & 71.08 & 394.46 & 81.17 & $\Delta H_{\mathrm{f}}$ Run & \\
\hline & & 375.63 & 77.61 & 404.11 & 82.74 & 386.37 & 80.41 \\
\hline & & 379.76 & 126.6 & 413.77 & 83.95 & 390.30 & 80.66 \\
\hline \multicolumn{8}{|c|}{ Pure Sample II-results using Mark II calorimetric cryostat } \\
\hline \multicolumn{2}{|c|}{ Series VIII } & 16.73 & 3.173 & 93.33 & 18.14 & \multicolumn{2}{|c|}{ Series XI } \\
\hline 131.10 & 23.88 & 18.69 & 3.742 & & & 207.40 & 37.25 \\
\hline 139.81 & 25.32 & 20.80 & 4.343 & \multirow{2}{*}{\multicolumn{2}{|c|}{ Series X }} & 217.25 & 39.08 \\
\hline & & 23.16 & 4.992 & & & 227.09 & 41.00 \\
\hline \multirow{2}{*}{\multicolumn{2}{|c|}{ Series IX }} & 25.74 & 5.673 & 99.27 & 18.99 & 236.85 & 42.91 \\
\hline & & 28.67 & 6.420 & 107.10 & 20.14 & 246.55 & 44.78 \\
\hline 4.87 & 0.151 & 31.61 & 7.136 & 116.32 & 21.54 & 256.29 & 46.69 \\
\hline 5.50 & 0.220 & 34.84 & 7.903 & 126.12 & 23.09 & 265.83 & 48.60 \\
\hline 6.17 & 0.307 & 39.09 & 8.847 & 136.02 & 24.69 & 275.30 & 50.44 \\
\hline 6.79 & 0.394 & 43.54 & 9.777 & 145.67 & 26.27 & 285.37 & 52.44 \\
\hline 7.58 & 0.580 & 47.78 & 10.63 & 155.19 & 27.88 & 295.63 & 54.50 \\
\hline 8.50 & 0.813 & 52.83 & 11.54 & 164.61 & 29.49 & $\begin{array}{l}295.03 \\
305.60\end{array}$ & 56.41 \\
\hline 9.46 & 1.111 & 58.31 & 12.57 & 174.04 & 31.12 & 315.34 & 58.39 \\
\hline 10.56 & 1.393 & 64.02 & 13.59 & 183.54 & 32.83 & 324.87 & 60.28 \\
\hline 11.82 & 1.703 & 69.94 & 14.53 & 193.07 & 34.54 & $\begin{array}{l}324.01 \\
334.16\end{array}$ & 6204 \\
\hline 13.24 & 2.134 & 76.58 & 15.56 & 202.50 & 36.33 & $\begin{array}{l}334.10 \\
34323\end{array}$ & $\begin{array}{l}02.04 \\
63.89\end{array}$ \\
\hline 14.89 & 2.630 & 84.51 & 16.85 & 212.06 & 38.13 & 349.23 & 05.09 \\
\hline
\end{tabular}


TABLE 2-continued

\begin{tabular}{|c|c|c|c|c|c|c|c|}
\hline$\frac{\langle T\rangle}{\mathrm{K}}$ & $\frac{C_{\mathrm{s}}}{\mathrm{cal} \mathrm{mol} \mathrm{l}^{-1} \mathrm{~K}^{-1}}$ & $\frac{\langle T\rangle}{\mathrm{K}}$ & $\frac{C_{\mathrm{s}}}{\text { cal mol }{ }^{-1} \mathrm{~K}^{-1}}$ & $\frac{\langle T\rangle}{\mathrm{K}}$ & $\frac{C_{\mathrm{s}}}{\mathrm{cal} \mathrm{mol}^{-1} \mathrm{~K}^{-1}}$ & $\frac{\langle T\rangle}{\mathbf{K}}$ & $\frac{C_{\mathrm{s}}}{\mathrm{cal} \mathrm{mol}^{-1} \mathrm{~K}^{-1}}$ \\
\hline \multicolumn{8}{|c|}{ Pure Sample II-results using Mark IV calorimetric thermostat } \\
\hline \multicolumn{2}{|c|}{ Series XII } & \multicolumn{2}{|c|}{ Series XIII } & 380.14 & 74.45 & \multicolumn{2}{|c|}{$\Delta H_{\mathrm{e}} \operatorname{Run} \mathrm{A}$} \\
\hline 296.53 & 54.33 & 295.98 & 54.20 & 382.55 & 752.9 & \multirow{3}{*}{\multicolumn{2}{|c|}{ Series XV }} \\
\hline 304.76 & 56.45 & 304.91 & 56.47 & 383.18 & 9734 & & \\
\hline 313.93 & 58.59 & 314.41 & 58.46 & 383.25 & 21590 & & \\
\hline 323.37 & 60.45 & 323.97 & 60.39 & 383.28 & 35270 & 365.22 & 68.41 \\
\hline 332.92 & 62.26 & 333.39 & 62.20 & 383.31 & 36390 & 374.39 & 71.17 \\
\hline 342.45 & 64.02 & 342.73 & 64.06 & 387.01 & 98.44 & $\Delta H_{\mathrm{f}} \mathrm{Ru}$ & in $\mathrm{B}$ \\
\hline 351.95 & 66.25 & 352.02 & 65.89 & 394.97 & 81.52 & 393.69 & 81.39 \\
\hline 361.39 & 68.06 & 361.27 & 67.50 & \multirow{2}{*}{\multicolumn{2}{|c|}{ Series XIV }} & 403.27 & 82.69 \\
\hline 370.85 & 69.96 & 369.55 & 69.29 & & & 412.97 & 84.15 \\
\hline & & 375.76 & 70.67 & $\Delta H \mathrm{Ru}$ & & 422.57 & 85.88 \\
\hline \multicolumn{8}{|c|}{ Triphenylene $\left(\mathrm{C}_{18} \mathrm{H}_{12}\right)$} \\
\hline \multicolumn{8}{|c|}{ Results using Mark II calorimetric cryostat } \\
\hline \multicolumn{2}{|r|}{ Series I } & 333.61 & 69.74 & \multicolumn{2}{|c|}{ Series III } & 29.13 & 6.695 \\
\hline 182.01 & 36.75 & \multirow{2}{*}{\multicolumn{2}{|c|}{ Series II }} & 4.88 & 0.104 & 32.89 & 7.804 \\
\hline 190.83 & 38.57 & & & 5.32 & 0.135 & 37.15 & 8.999 \\
\hline 199.80 & 40.44 & 78.89 & 17.75 & 5.98 & 0.202 & \multirow{2}{*}{\multicolumn{2}{|c|}{ Series IV }} \\
\hline 209.05 & 42.37 & 89.30 & 19.64 & 6.80 & 0.300 & & \\
\hline 218.65 & 44.43 & 98.52 & 21.16 & 7.59 & 0.429 & 37.27 & 9.030 \\
\hline 228.37 & 46.55 & 107.16 & 22.65 & 8.40 & 0.603 & 41.50 & 10.11 \\
\hline 237.98 & 48.62 & 116.19 & 24.25 & 9.32 & 0.836 & 46.20 & 11.25 \\
\hline 247.47 & 50.70 & 125.33 & 25.91 & 10.41 & 1.105 & 51.20 & 12.38 \\
\hline 257.05 & 52.86 & 134.42 & 27.60 & 11.75 & 1.419 & 56.43 & 13.49 \\
\hline 266.72 & 54.96 & 143.79 & 29.35 & 13.36 & 1.873 & 62.28 & 14.69 \\
\hline 276.10 & 57.05 & 152.99 & 31.04 & 15.11 & 2.394 & & \\
\hline 285.42 & 59.12 & 162.21 & 32.85 & 16.98 & 2.962 & \multirow{2}{*}{\multicolumn{2}{|c|}{ Series V }} \\
\hline 295.07 & 61.28 & 171.46 & 34.69 & 19.02 & 3.591 & & \\
\hline 304.77 & 63.44 & 180.75 & 36.58 & 21.05 & 4.223 & 60.70 & 14.37 \\
\hline 314.42 & 65.58 & 190.04 & 38.46 & 23.22 & 4.896 & 67.62 & 15.70 \\
\hline 324.00 & 67.68 & & & 25.90 & 5.722 & 74.96 & 17.00 \\
\hline \multicolumn{8}{|c|}{ Results using Mark IV calorimetric thermostat } \\
\hline \multicolumn{2}{|c|}{ Series VI } & \multicolumn{2}{|c|}{ Series VII } & 473.14 & 264.3 & \multicolumn{2}{|c|}{ Series IX } \\
\hline 316.59 & 65.90 & 391.77 & 81.71 & & & 451.81 & 92.92 \\
\hline 328.06 & 68.48 & 403.20 & 83.84 & \multirow{2}{*}{\multicolumn{2}{|c|}{ Series VIII }} & 460.22 & 94.62 \\
\hline 339.22 & 70.84 & 414.38 & 86.10 & & & \multicolumn{2}{|c|}{$\Delta H_{\mathrm{f}}$ Run $\mathrm{A}$} \\
\hline 350.55 & 73.25 & 425.33 & 88.21 & 458.88 & 94.25 & & \\
\hline 362.03 & 75.69 & 436.06 & 90.27 & 466.41 & 99.53 & \multirow{2}{*}{\multicolumn{2}{|c|}{ Series X }} \\
\hline 373.23 & 78.11 & 446.58 & 92.46 & 470.47 & 1222 & & \\
\hline 384.18 & 80.15 & 456.93 & 94.18 & 470.88 & 14950 & 463.14 & 95.05 \\
\hline 395.34 & 82.44 & 466.44 & 115.3 & 470.93 & 26250 & $\Delta H_{\mathrm{f}} \mathrm{Ru}$ & $\ln B$ \\
\hline 406.71 & 84.52 & 470.87 & 8019 & 470.96 & 66790 & 481.02 & 106.5 \\
\hline \multirow[t]{3}{*}{418.01} & 86.93 & 470.94 & 17770 & 470.97 & 68680 & 490.27 & 107.5 \\
\hline & & 470.96 & 37980 & 470.981 & 306000 & 499.78 & 108.7 \\
\hline & & 470.98 & 20730 & 473.32 & 173.6 & 509.55 & 110.4 \\
\hline
\end{tabular}


TABLE 3. Thermodynamic properties of pyrene, fluoranthene, and triphenylene

\begin{tabular}{|c|c|c|c|c|}
\hline$\frac{T}{\mathrm{~K}}$ & $\frac{C_{\mathrm{s}}}{\text { cal } \mathrm{mol}^{-1} \mathrm{~K}^{-1}}$ & $\frac{S^{\circ}}{\text { cal mol }{ }^{-1} \mathrm{~K}^{-1}}$ & $\frac{H^{\circ}-H_{0}^{\circ}}{\text { cal } \mathrm{mol}^{-1}}$ & $\frac{-\left(G^{\circ}-H_{0}^{\circ}\right) / T}{\text { cal } \mathrm{mol}^{-1} \mathrm{~K}^{-1}}$ \\
\hline $\begin{array}{c}\text { Crystal II phase } \\
5 \\
10 \\
15 \\
20 \\
25 \\
30 \\
35 \\
40 \\
45 \\
50 \\
60 \\
70 \\
80 \\
90 \\
100 \\
110\end{array}$ & $\begin{array}{c}0.159 \\
1.163 \\
2.618 \\
4.053 \\
5.383 \\
6.587 \\
7.687 \\
8.688 \\
9.593 \\
10.46 \\
12.07 \\
13.57 \\
15.05 \\
16.53 \\
17.96 \\
19.46\end{array}$ & $\begin{array}{c}\text { Pyrene }\left(\mathrm{C}_{16} \mathrm{H}_{\mathbf{1 0}}\right) \\
0.054 \\
0.413 \\
1.157 \\
2.109 \\
3.159 \\
4.248 \\
5.348 \\
6.441 \\
7.517 \\
8.572 \\
10.62 \\
12.60 \\
14.51 \\
16.36 \\
18.18 \\
19.96\end{array}$ & $\begin{array}{c}0.202 \\
3.085 \\
12.51 \\
29.22 \\
52.86 \\
82.84 \\
118.6 \\
159.5 \\
205.3 \\
255.4 \\
368.2 \\
496.4 \\
639.4 \\
797.4 \\
969.8 \\
1157\end{array}$ & $\begin{array}{l}0.014 \\
0.105 \\
0.324 \\
0.648 \\
1.044 \\
1.487 \\
1.960 \\
2.452 \\
2.955 \\
3.464 \\
4.488 \\
5.506 \\
6.512 \\
7.504 \\
8.481 \\
9.444\end{array}$ \\
\hline $\begin{array}{c}\text { Transition region } \\
115 \\
120 \\
120.75 \\
125 \\
130 \\
135\end{array}$ & $\begin{array}{l}20.45 \\
27.20 \\
33.30 \\
26.58 \\
24.08 \\
23.67\end{array}$ & $\begin{array}{l}20.84 \\
21.79 \\
21.98 \\
23.01 \\
23.99 \\
24.89\end{array}$ & $\begin{array}{l}1257 \\
1369 \\
1392 \\
1517 \\
1643 \\
1762\end{array}$ & $\begin{array}{r}9.91 \\
10.38 \\
10.45 \\
10.87 \\
11.35 \\
11.84\end{array}$ \\
\hline $\begin{array}{c}\text { Crystal I phase } \\
140 \\
150 \\
160 \\
170 \\
180 \\
190 \\
200 \\
210 \\
220 \\
230 \\
240 \\
250 \\
260 \\
270 \\
273.15 \\
280 \\
290 \\
298.15 \\
300 \\
310 \\
320 \\
330 \\
340 \\
350 \\
360\end{array}$ & $\begin{array}{l}24.28 \\
25.97 \\
27.68 \\
29.41 \\
31.19 \\
33.01 \\
34.89 \\
36.81 \\
38.78 \\
40.78 \\
42.81 \\
44.86 \\
46.92 \\
48.99 \\
49.65 \\
51.07 \\
53.17 \\
54.90 \\
55.30 \\
57.46 \\
59.66 \\
61.92 \\
64.24 \\
66.63 \\
69.09\end{array}$ & $\begin{array}{l}25.76 \\
27.49 \\
29.22 \\
30.95 \\
32.68 \\
34.42 \\
36.16 \\
37.91 \\
39.66 \\
41.43 \\
43.21 \\
45.00 \\
46.80 \\
48.61 \\
49.18 \\
50.43 \\
52.26 \\
53.75 \\
54.10 \\
55.94 \\
57.80 \\
59.67 \\
61.56 \\
63.45 \\
65.36\end{array}$ & $\begin{array}{r}1881 \\
2133 \\
2400 \\
2686 \\
2989 \\
3310 \\
3650 \\
4008 \\
4386 \\
4784 \\
5202 \\
5640 \\
6099 \\
6578 \\
6734 \\
7079 \\
7600 \\
8040 \\
8142 \\
8706 \\
9291 \\
9899 \\
10530 \\
11180 \\
11860\end{array}$ & $\begin{array}{l}12.32 \\
13.27 \\
14.22 \\
15.15 \\
16.08 \\
17.00 \\
17.91 \\
18.82 \\
19.73 \\
20.63 \\
21.54 \\
22.44 \\
23.34 \\
24.24 \\
24.53 \\
25.15 \\
26.05 \\
26.79 \\
26.95 \\
27.86 \\
28.77 \\
29.67 \\
30.58 \\
31.50 \\
32.41\end{array}$ \\
\hline
\end{tabular}


TABLE 3-continued

\begin{tabular}{|c|c|c|c|c|}
\hline$\frac{T}{\mathbf{K}}$ & $\frac{C_{\mathrm{s}}}{\mathrm{cal} \mathrm{mol} \mathbf{m}^{-1} \mathrm{~K}^{-1}}$ & $\frac{S^{\circ}}{\mathrm{cal} \mathrm{mol} \mathrm{m}^{-1} \mathrm{~K}^{-1}}$ & $\frac{H^{\circ}-H_{0}^{\circ}}{\text { cal mol }^{-1}}$ & $\frac{-\left(G^{\circ}-H_{0}^{\circ}\right) / T}{\mathrm{cal} \mathrm{mol}^{-1} \mathrm{~K}^{-1}}$ \\
\hline \multicolumn{5}{|c|}{ Crystal I Phase-continued } \\
\hline $\begin{array}{l}370 \\
380 \\
390 \\
400 \\
410 \\
423.81^{a}\end{array}$ & $\begin{array}{c}71.65 \\
74.33 \\
77.20 \\
80.35 \\
83.96 \\
\mathbf{( 8 8 . 9 3 )}\end{array}$ & $\begin{array}{l}67.29 \\
69.24 \\
71.20 \\
73.20 \\
75.22 \\
78.08\end{array}$ & $\begin{array}{l}12570 \\
13300 \\
14050 \\
14840 \\
15660 \\
16850\end{array}$ & $\begin{array}{l}33.33 \\
34.25 \\
35.17 \\
36.09 \\
37.02 \\
38.32\end{array}$ \\
\hline \multicolumn{5}{|l|}{ Liquid phase } \\
\hline $\begin{array}{l}423.81^{a} \\
430 \\
440 \\
450 \\
460 \\
470 \\
480\end{array}$ & $\begin{array}{c}(83.34) \\
84.16 \\
85.56 \\
86.89 \\
88.19 \\
89.52 \\
90.95\end{array}$ & $\begin{array}{l}87.87 \\
89.17 \\
91.12 \\
93.06 \\
94.98 \\
96.89 \\
98.79\end{array}$ & $\begin{array}{l}21000 \\
21560 \\
22400 \\
23270 \\
24140 \\
25030 \\
25930\end{array}$ & $\begin{array}{l}38.32 \\
39.04 \\
40.20 \\
41.36 \\
42.50 \\
43.64 \\
44.77\end{array}$ \\
\hline \multicolumn{5}{|c|}{ Fluoranthene $\left(\mathrm{C}_{16} \mathrm{H}_{10}\right)$} \\
\hline 5 & 0.162 & 0.056 & 0.211 & 0.014 \\
\hline 10 & 1.219 & 0.436 & 3.255 & 0.110 \\
\hline 15 & 2.663 & 1.202 & 12.94 & 0.339 \\
\hline 20 & 4.107 & 2.166 & 29.88 & 0.672 \\
\hline 25 & 5.488 & 3.233 & 53.91 & 1.076 \\
\hline 30 & 6.750 & 4.347 & 84.55 & 1.528 \\
\hline 35 & 7.935 & 5.4 .77 & 121.3 & 2.012 \\
\hline 40 & 9.040 & 6.610 & 163.8 & 2.516 \\
\hline 45 & 10.08 & 7.74 & 211.6 & 3.033 \\
\hline 50 & 11.06 & 8.85 & 264.4 & 3.559 \\
\hline 60 & 12.88 & 11.03 & 384.2 & 4.623 \\
\hline 70 & 14.55 & 13.14 & 521.5 & 5.690 \\
\hline 80 & 16.12 & 15.19 & 674.9 & 6.749 \\
\hline 90 & 17.63 & 17.17 & 843.7 & 7.798 \\
\hline 100 & 19.10 & 19.11 & 1027 & 8.832 \\
\hline 110 & 20.60 & 21.00 & 1226 & 9.852 \\
\hline 120 & 22.13 & 22.85 & 1439 & 10.86 \\
\hline 130 & 23.70 & 24.69 & 1669 & 11.85 \\
\hline 140 & 25.33 & 26.50 & 1914 & 12.83 \\
\hline 150 & 27.00 & 28.31 & 2175 & 13.80 \\
\hline 160 & 28.70 & 30.10 & 2454 & 14.77 \\
\hline 170 & 30.43 & 31.89 & 2749 & 15.72 \\
\hline 180 & 32.19 & 33.68 & 3062 & 16.67 \\
\hline 190 & 34.00 & 35.47 & 3393 & 17.61 \\
\hline 200 & 35.84 & 37.26 & 3742 & 18.55 \\
\hline 210 & 37.72 & 39.06 & 4110 & 19.48 \\
\hline 220 & 39.63 & 40.86 & 4497 & 20.41 \\
\hline 230 & 41.57 & 42.66 & 4903 & 21.34 \\
\hline 240 & 43.52 & 44.47 & 5328 & 22.27 \\
\hline 250 & 45.47 & 46.29 & 5773 & 23.19 \\
\hline
\end{tabular}


TABLE 3-continued

\begin{tabular}{|c|c|c|c|c|}
\hline$\frac{T}{\mathrm{~K}}$ & 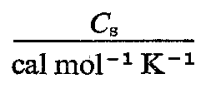 & $\frac{S^{o}}{\mathrm{cal} \mathrm{mol}^{-1} \mathrm{~K}^{-1}}$ & $\frac{H^{\circ}-H_{0}^{\circ}}{\mathrm{cal} \mathrm{mol}^{-1}}$ & $\frac{-\left(G^{\circ}-H_{0}^{\circ}\right) / T}{\mathrm{cal} \mathrm{mol}^{-1} \mathrm{~K}^{-1}}$ \\
\hline \multicolumn{5}{|c|}{ Crystal I phase-continued } \\
\hline $\begin{array}{l}260 \\
270 \\
273.15 \\
280 \\
290 \\
298.15 \\
300 \\
310 \\
320 \\
330 \\
340 \\
350 \\
360 \\
370 \\
380 \\
383.36^{a}\end{array}$ & $\begin{array}{c}47.43 \\
49.39 \\
50.02 \\
51.38 \\
53.38 \\
55.03 \\
55.40 \\
57.42 \\
59.43 \\
61.42 \\
63.40 \\
65.37 \\
67.34 \\
69.38 \\
71.42 \\
(72.08)\end{array}$ & $\begin{array}{l}48.11 \\
49.93 \\
50.51 \\
51.77 \\
53.60 \\
55.11 \\
55.45 \\
57.30 \\
59.15 \\
61.01 \\
62.87 \\
64.74 \\
66.61 \\
68.48 \\
70.36 \\
70.92\end{array}$ & $\begin{array}{r}6238 \\
6722 \\
6878 \\
7226 \\
7749 \\
8191 \\
8293 \\
8857 \\
9442 \\
10046 \\
10670 \\
11314 \\
11980 \\
12660 \\
13370 \\
13580\end{array}$ & $\begin{array}{l}24.12 \\
25.04 \\
25.33 \\
25.96 \\
26.88 \\
27.63 \\
27.80 \\
28.73 \\
29.65 \\
30.57 \\
31.49 \\
32.42 \\
33.34 \\
34.26 \\
35.19 \\
35.50\end{array}$ \\
\hline \multicolumn{5}{|l|}{ Liquid phase } \\
\hline $\begin{array}{l}383.36^{a} \\
390 \\
400 \\
410 \\
420\end{array}$ & $\begin{array}{l}(79.97) \\
80.83 \\
82.21 \\
83.74 \\
85.42\end{array}$ & $\begin{array}{l}82.61 \\
84.06 \\
86.12 \\
88.17 \\
90.21\end{array}$ & $\begin{array}{l}18057 \\
18620 \\
19430 \\
20260 \\
21110\end{array}$ & $\begin{array}{l}35.50 \\
36.32 \\
37.54 \\
38.75 \\
39.95\end{array}$ \\
\hline \multicolumn{5}{|c|}{ Triphenylene $\left(\mathrm{C}_{16} \mathrm{H}_{10}\right)$} \\
\hline $\begin{array}{r}5 \\
10 \\
15 \\
20 \\
25 \\
30 \\
35 \\
40 \\
45 \\
50 \\
60 \\
70 \\
80 \\
90 \\
100 \\
110 \\
120 \\
130 \\
140 \\
150 \\
160 \\
170 \\
180 \\
190\end{array}$ & $\begin{array}{r}0.113 \\
0.979 \\
2.364 \\
3.890 \\
5.449 \\
6.958 \\
8.398 \\
9.732 \\
10.97 \\
12.12 \\
14.23 \\
16.14 \\
17.93 \\
19.69 \\
21.43 \\
23.19 \\
24.96 \\
26.76 \\
28.60 \\
30.48 \\
32.41 \\
34.39 \\
36.40 \\
38.44\end{array}$ & $\begin{array}{c}0.038 \\
0.327 \\
0.978 \\
1.865 \\
2.901 \\
4.029 \\
5.210 \\
6.421 \\
7.639 \\
8.855 \\
11.26 \\
13.59 \\
15.87 \\
18.08 \\
20.25 \\
22.37 \\
24.46 \\
26.53 \\
28.58 \\
30.62 \\
32.65 \\
34.67 \\
36.70 \\
38.72\end{array}$ & $\begin{array}{c}0.142 \\
2.474 \\
10.74 \\
26.34 \\
49.69 \\
80.74 \\
119.2 \\
164.6 \\
216.3 \\
274.1 \\
406.1 \\
558.1 \\
728.5 \\
916.6 \\
1122 \\
1345 \\
1586 \\
1845 \\
2121 \\
2417 \\
2731 \\
3065 \\
3419 \\
3793\end{array}$ & $\begin{array}{r}0.010 \\
0.079 \\
0.262 \\
0.548 \\
0.913 \\
1.337 \\
1.806 \\
2.307 \\
2.832 \\
3.373 \\
4.488 \\
5.622 \\
6.762 \\
7.897 \\
9.024 \\
10.14 \\
11.25 \\
12.34 \\
13.43 \\
14.51 \\
15.58 \\
16.64 \\
17.70 \\
18.75\end{array}$ \\
\hline
\end{tabular}


TABLE 3-continued

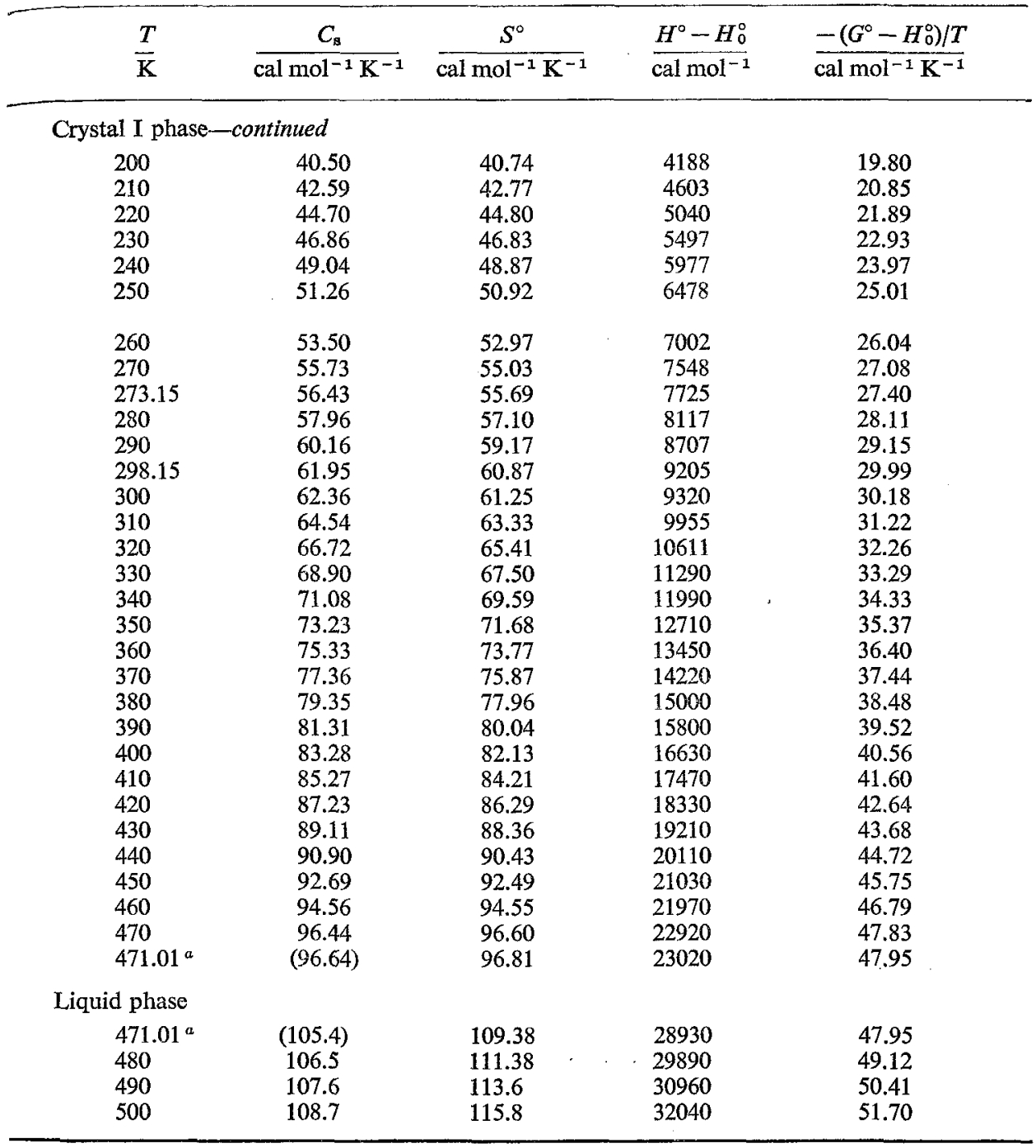

a Assuming melting to be truly isothermal.

sequence so that the approximate temperature increments employed in the measurements may usually be deduced from the differences in the adjacent mean temperatures. Where appropriate, the values have been corrected for curvature, i.e., for the difference between the ratio of finite increments to the differential relation. Vaporization corrections are insignificant.

It is believed that the precision of these results is reflected by probable errors decreasing from 5 per cent at $5 \mathrm{~K}$ to 1 per cent at $15 \mathrm{~K}$ and to less than 0.1 per cent at higher temperatures. Values of the smooth heat capacities at saturation $C_{\mathrm{s}}$ obtained 
from digital-computer fits agree well with those read from large-scale plots of the results and are presented in table 3 at selected temperatures, together with the entropy $S^{\circ}$, enthalpy increment $\left(H^{\circ}-H_{0}^{\circ}\right)$, and Gibbs energy function $-\left(G^{\circ}-H_{0}^{\circ}\right) / T$. These properties were obtained by fitting a polynomial through the results by the method of least-squares and integrating the resulting functions. Below $5 \mathrm{~K}$, the functions were estimated from the Debye $T^{3}$ limiting law by plotting $C_{\mathrm{s}} / T$ against $T^{2}$. The probable errors of the values in table 3 are considered to be less than 0.1 per cent above $100 \mathrm{~K}$. Nuclear spin and isotopic mixing effects have not been incorporated in the evaluation of the entropy and Gibbs energy function so that these values are practical for use in chemical equilibrium calculations.

\section{PYRENE-TRANSITION}

The heat capacity of pyrene was previously reported by Jacobs and Parks ${ }^{(4)}$ from 90 to $300 \mathrm{~K}$ with an assigned "estimated reliability of \pm 1 per cent." Their points do indeed scatter over most of the common region by approximately this amount from the present results, except above $260 \mathrm{~K}$ where, as may be noted in figure 2 , they are about 1.5 per cent higher than the present result.

In the present measurements, a thermal anomaly is found with a maximum at $120.8 \mathrm{~K}$, a temperature slightly higher than the value $(116 \mathrm{~K})$ at which Jacobs and Parks noted a hump in the heat capacity. The cause of the anomaly has been attributed to a slight change in the lattice structure. Powder photographs taken at room temperature and at liquid-air temperature ${ }^{(11)}$ are interpreted as indicating the

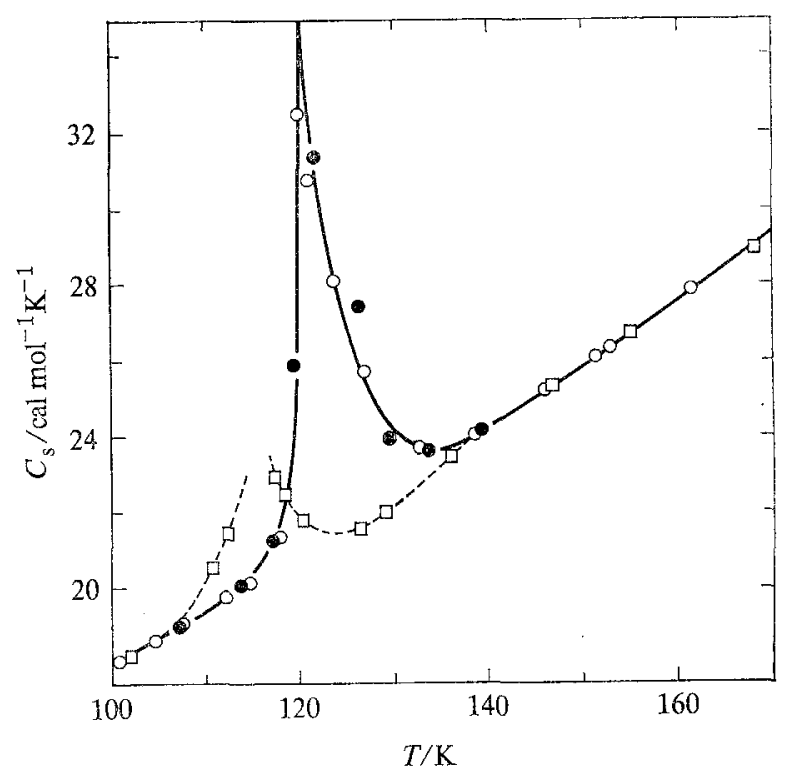

FIGURE 2. The heat capacity of pyrene in the transition region. The points and curves indicated $-\mathrm{O}-\mathrm{-}$ - are from the present research; those shown $--\square--$ are from Jacobs and Parks. ${ }^{(7)}$ 
TABLE 4. Enthalpy increments of transition and melting for pyrene and for melting of fluoranthene and triphenylene

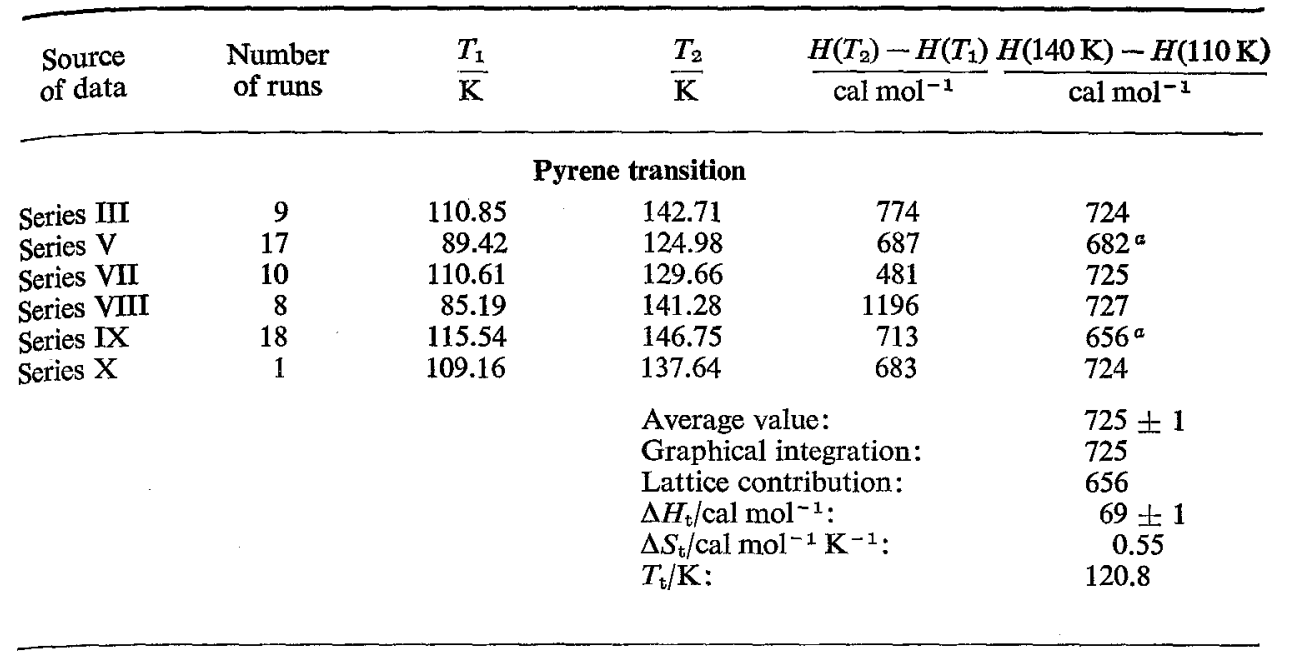

\begin{tabular}{|c|c|c|c|c|c|}
\hline $\begin{array}{l}\text { Source } \\
\text { of data }\end{array}$ & $\begin{array}{l}\text { Number } \\
\text { of runs }\end{array}$ & $\frac{T_{1}}{\mathrm{~K}}$ & $\frac{T_{2}}{\mathrm{~K}}$ & $H\left(T_{2}\right)-H\left(T_{1}\right)$ & $\frac{H(430 \mathrm{~K})-H(410 \mathrm{~K})}{\mathrm{cal} \mathrm{mol}-1}$ \\
\hline \multicolumn{6}{|c|}{ Pyrene melting } \\
\hline $\begin{array}{l}\text { Series XI } \\
\text { Series XII } \\
\text { Series XV } \\
\text { Series XVI }\end{array}$ & $\begin{array}{l}6 \\
8 \\
1 \\
1\end{array}$ & $\begin{array}{l}409.52 \\
413.47 \\
413.94 \\
420.78\end{array}$ & $\begin{array}{l}428.84 \\
429.13 \\
431.60 \\
425.55\end{array}$ & $\begin{array}{l}5842 \\
5555 \\
5694 \\
4581\end{array}$ & $\begin{array}{l}5900 \\
5896 \\
5894 \\
5894\end{array}$ \\
\hline \multicolumn{5}{|c|}{$\begin{array}{l}\text { Average value: } \\
\text { Graphical integration: } \\
\text { Lattice contribution: } \\
\Delta H_{\mathrm{m}} / \mathrm{cal} \mathrm{mol}^{-1}: \\
\Delta S_{\mathrm{m}} / \mathrm{cal} \mathrm{mol}^{-1} \mathrm{~K}^{-1}: \\
T_{\mathrm{m}} / \mathrm{K}:\end{array}$} & $\begin{array}{l}5896 \pm 1 \\
5894 \\
1746 \\
4150 \pm 1 \\
9.79 \\
423.81\end{array}$ \\
\hline Source & Number & $T_{1}$ & $T_{2}$ & $H\left(T_{2}\right)-H\left(T_{1}\right)$ & $H(385 \mathrm{~K})-H(370 \mathrm{~K})$ \\
\hline of data & of runs & $\overrightarrow{\mathrm{K}}$ & $\overline{\mathbf{K}}$ & $\mathrm{cal} \mathrm{mol}^{-1}$ & cal mol-1 \\
\hline \multicolumn{6}{|c|}{ Impure fluoranthene (Sample D melting } \\
\hline Series X & 6 & 377.62 & 386.64 & 5078 & 5513 \\
\hline Series XII & 7 & 373.64 & 389.12 & 5607 & 5536 \\
\hline Series XIII & 1 & 370.79 & 389.68 & 5852 & 5531 \\
\hline Series XIV & 1 & 378.42 & 391.15 & 5384 & 5520 \\
\hline Series XV & 1. & 374.66 & 384.91 & 5179 & 5523 \\
\hline \multicolumn{5}{|c|}{$\begin{array}{l}\text { Average value: } \\
\text { Lattice contribution: } \\
\Delta H_{\mathrm{m}}(\text { impure }) / \mathrm{cal} \mathrm{mol}^{-1} \text { : }\end{array}$} & $\begin{array}{l}5525 \pm 4 \\
1067 \\
4458 \pm 4\end{array}$ \\
\hline
\end{tabular}


TABLE 4-continued

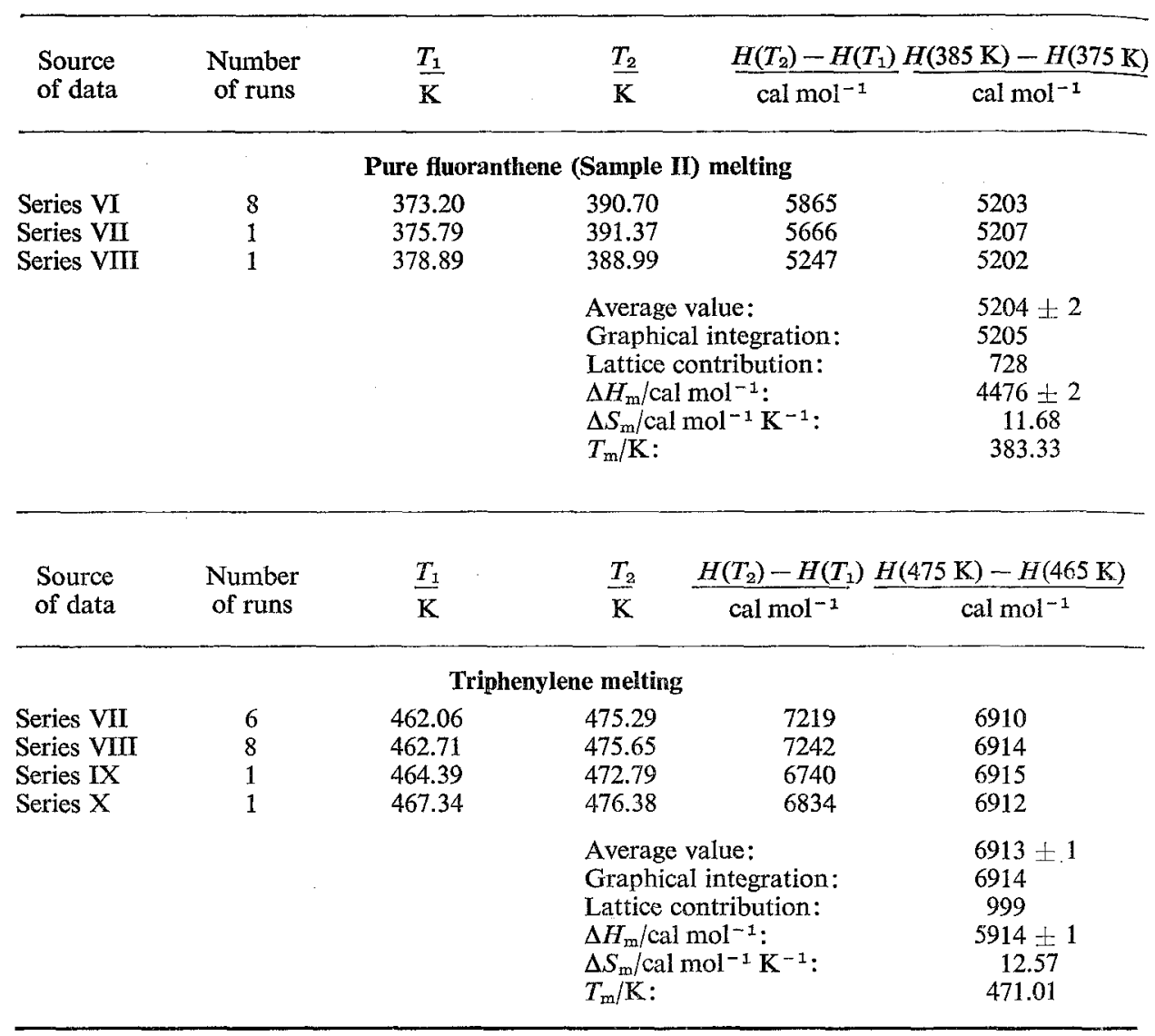

${ }^{a}$ Excluded from the average because rapidity of cooling obviously occasioned same undercooling of the Crystal I phase.

presence of such a phase transformation at an intermediate temperature. Seven sets of heat-capacity and enthalpy-type determinations (summarized in table 4) were made through the anomalous region, and the excess enthalpy (of transformation) was obtained by subtracting the interpolated lattice contribution from the total. The entropy contribution was integrated from a smooth curve drawn through points from several series of heat-capacity determinations through the anomalous region and constrained to give the proper total enthalpy. As shown in table 4, the corresponding values are $68.8 \mathrm{cal} \mathrm{mol}^{-1}$ for the enthalpy increment, compared with $24 \mathrm{cal} \mathrm{mol}^{-1}$ obtained in the previous study. ${ }^{(4)}$ These differences are attributed to the higher purity of the present sample, since Jacobs and Parks purified their pyrene only by recrystallization from benzene and obtained light yellow-colored crystals. The transformational excess entropy is estimated to be $0.55 \mathrm{cal} \mathrm{mol}^{-1} \mathrm{~K}^{-1}$. 
PYRENE-MELTING

The four sets of determinations made through the melting region are also summarized in table 4. Removal of the extrapolated contributions of the crystalline and liquid phases yield a value for the enthalpy of melting, $\Delta H_{\mathrm{m}}=4148.9 \mathrm{cal} \mathrm{mol}^{-1}$ and a corresponding entropy of melting, $\Delta S_{\mathrm{m}}=9.79 \mathrm{cal} \mathrm{mol}^{-1} \mathrm{~K}^{-1}$. The amount of solidsoluble, liquid-soluble impurity was estimated from the fractional melting results of table 5. The triple point of the experimental sample was found to be $423.78 \mathrm{~K}$ and that of the pure substance to be $423.81 \mathrm{~K}$. Previously reported melting temperatures are $148^{\circ} \mathrm{C}^{(12)}$ and $151^{\circ} \mathrm{C} .{ }^{(13)}$ The slope of the fractional fusion curve corresponds to 0.0003 mole fraction of liquid-soluble, solid-soluble impurity on the basis of the van't Hoff equation.

\section{FLUORANTHENE-HEAT CAPACITY AND MELTING}

The initial measurements were made on Impure Sample I which had been sublimed but not zone-melted. A distinct pre-melting effect and an excess slope of the melting curve were found. The curvature of the plot of $T$ against $1 / F$ suggested that impurity was present also in solid solution. Applications of the treatment of Mastrangelo and Dornte ${ }^{(14)}$ for solid-soluble, liquid-soluble contaminants indicated 0.0039 mole fraction of impurity and a ratio of solubility between the solid and liquid phases of 0.0883 . The triple point of the pure compound was extrapolated as $383.34 \mathrm{~K}$. After zone-refining the material, the new Pure Sample II (on which the definitive measurements were made) exhibited sharper melting.

Results through the melting region are presented in table 4 for three series of measurements on Sample I and for five series of measurements on Sample II, including one and two series of fractional melting results respectively. Because the sample is now sufficiently pure ( 0.00064 mole fraction impurity), the melting temperature may be treated in terms of the van't Hoff equation and yields extrapolated triple points of $383.29 \mathrm{~K}$ for Sample II and $383.33 \mathrm{~K}$ for the pure substance. The latter is in good agreement with the previous value on the impure material and with the reported value of $110^{\circ} \mathrm{C} .^{(15)}$ No significant differences in the heat capacity are observed from 300 to $350 \mathrm{~K}$ (within $33 \mathrm{~K}$ of melting) between the two samples.

In the evaluation of the enthalpies of melting, the same extrapolations were used to represent the lattice and liquid heat capacities for both samples. The mean $\Delta H_{\mathrm{m}}$ of $4477 \mathrm{cal} \mathrm{mol}^{-1}$ was calculated from the excess enthalpy of Sample II. The corresponding entropy increment of melting is $11.68 \mathrm{cal} \mathrm{mol}^{-1} \mathrm{~K}^{-1}$. A value of $4457 \mathrm{cal} \mathrm{mol}^{-1}$ was obtained for $\Delta H_{\mathrm{m}}$ on Sample I. If the value for the enthalpy of melting of Sample I is multiplied by the factor $1 / 0.99611$ to correct for the presence of only 0.99611 mole fraction of pure compound in this sample, the result is $4475 \mathrm{cal} \mathrm{mol}^{-1}$, in good accord with that found for the purer sample.

\section{TRIPHENYLENE-MELTING}

Apart from fusion, no thermal anomaly is found in the solid phase. The four series of measurements through the melting region are summarized in table 4 . The triple point of the pure material occurs at $471.01 \mathrm{~K}$ and at $470.98 \mathrm{~K}$ for the calorimetric 
TABLE 5. Fractional melting studies of pyrene, fluoranthene, and triphenylene

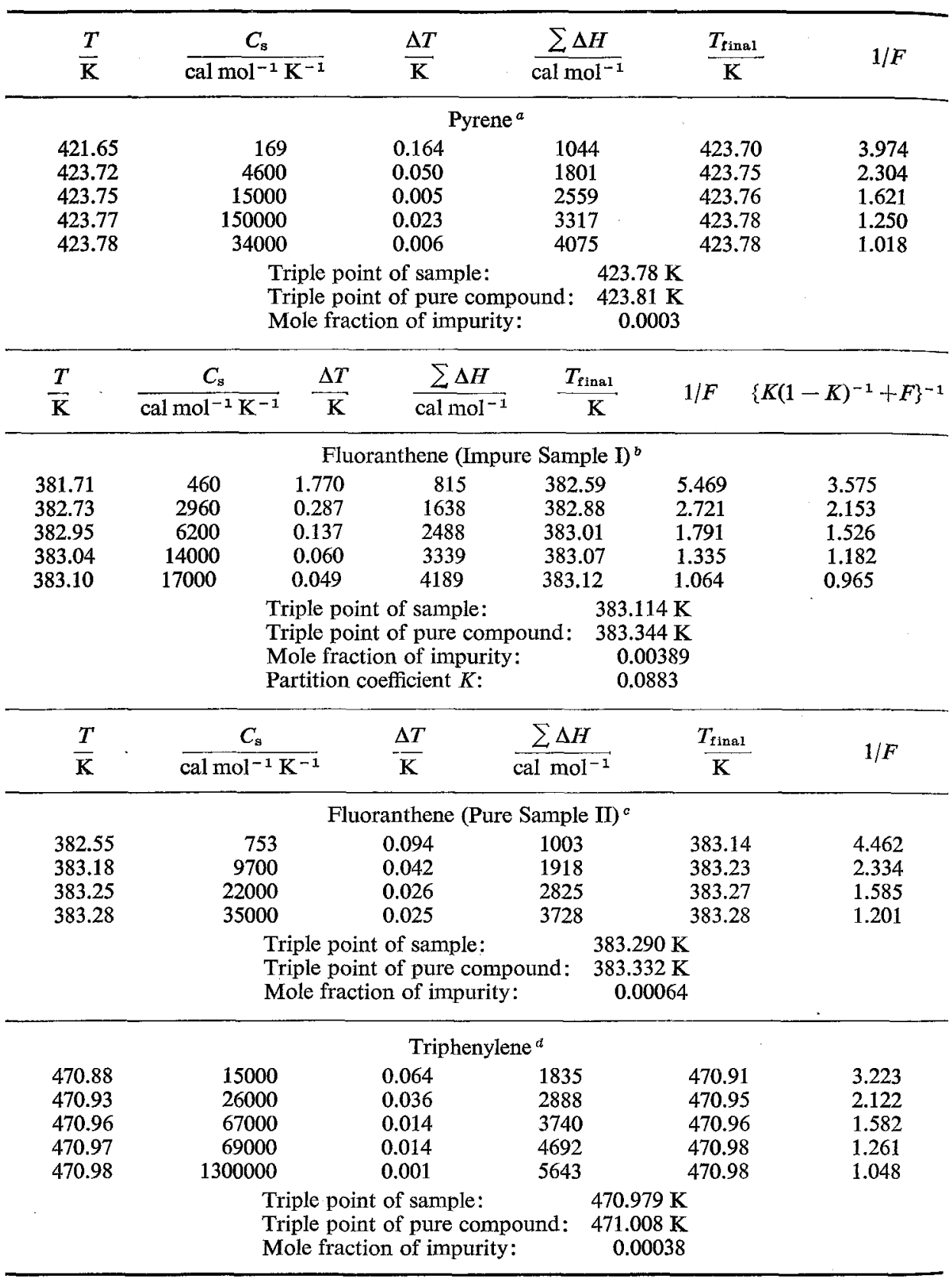

a Values from melting runs Series XII.

$b$ Values from melting runs Series XII.

$a$ Values from melting runs Series VI.

a Values from melting runs Series VIII. 
sample. Bachmann and Clarke ${ }^{(16)}$ report a melting temperature of $199^{\circ} \mathrm{C}$. The fractional melting data of table 5 indicate a solid-insoluble, liquid-soluble impurity of 0.00038 mole fraction. Melting occurs with a $\Delta H_{\mathrm{m}}$ of $5913 \mathrm{cal} \mathrm{mol}^{-1}$ and an entropy increment of $12.57 \mathrm{cal} \mathrm{mol}^{-1} \mathrm{~K}^{-1}$.

\section{Discussion}

\section{THE MECHANISM OF MELTING}

Although the main qualitative distinction between crystalline and liquid phases has long been attributed to the disappearance of the long-range order of the crystalline structure, as yet few theories are able to give satisfactory semi-quantitative descriptions of the thermodynamic changes involved. ${ }^{(17)}$ Presumably, positional, orientational (for non-spherically-symmetrical molecules), and configurational types of disorder are involved. In addition, increases in vibrational entropy appear to make significant contributions to the total entropy of fusion in certain crystal structures, such as those containing rigid polyatomic molecules of high anistropy.

Hitherto, melting studies have been available only for the simplest condensed polynuclear hydrocarbons; e.g. benzene, ${ }^{(18)}$ naphthalene, ${ }^{(19)}$ anthracene, and phenanthrene. ${ }^{(20)}$ The results of this study together with data on perylene ${ }^{(21)}$ provided entropies of fusion for four additional substances which are incorporated into table 6.

Since fused aromatic rings may be regarded as having an approximately planar repulsion envelope, they provide a useful range of shapes as a basis for the investigation of the structure of melts. For example, Ubbelohde and his collaborators $^{(11,22-24)}$ calculated the volume required for free rotation about principal axes for some condensed aromatic compounds on the basis of their van der Waals repulsion envelopes and compared these with the observed molar volumes. They concluded that most of the molecules studied do not have sufficient room to rotate freely (see table 6). However, Frank ${ }^{(25)}$ suggested that even when the volume per molecule in the liquid phase does not provide adequate space for rotation of individual molecules independently of their neighbors, two molecules may rotate "in gear" with each other. More than half of the rotational entropy survives because the effective combined rotator has twice the moment of inertia. Unless orientational disordering has already occurred in the crystals below $T_{\mathrm{m}}$, it is highly probable that orientational disordering will make important contributions to the total entropy of fusion. Generally, the expansion in volume on melting lowers the potential barriers opposing rotation.

Vibrational entropy changes upon fusion may also make a substantial contribution to the total entropy of melting of condensed polynuclear aromatic compounds. In the simplest theory for the vibrational entropy of a crystal containing $3 N$ vibrators, all having the same characteristic frequency $v_{\mathrm{c}}$, the increment on melting is $\Delta S_{\mathrm{vib}}=3 R \ln \left(v_{\mathrm{c}} / v_{1}\right)$ if $h v_{\mathrm{c}} \gg k T$, and the melt (l) can be treated as quasi-crystalline. The rigid planar molecules in these crystals may have significantly greater vibrational freedom in the liquid with values of $v_{1}$ considerably lower than $v_{\mathrm{c}}$. Part of the total 
TABLE 6. Thermodynamics of melting of polynuclear aromatic hydrocarbons

\begin{tabular}{|c|c|c|c|c|c|}
\hline Compound & Formula & $\frac{\Delta S_{\mathrm{m}}{ }^{a}}{\mathrm{cal} \mathrm{mol}^{-1} \mathrm{~K}^{-1}}$ & 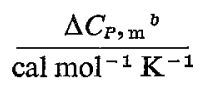 & $\frac{V(\mathrm{c}) / V_{\min }{ }^{c}}{\text { at } T_{\mathrm{m}}}$ & $\frac{V(1) / V_{\mathrm{m} 1 \mathrm{n}}{ }^{c, a}}{\text { at } T_{\mathrm{m}}}$ \\
\hline Benzene & $\mathrm{C}_{6} \mathrm{H}_{6}$ & $8.5^{e}$ & 0.7 & 0.94 & 1.06 \\
\hline Naphthalene & $\mathrm{C}_{1,0} \mathrm{H}_{8}$ & $12.8^{f}$ & $\approx 2$ & 0.89 & 1.05 \\
\hline Acenaphthene & $\mathrm{C}_{12} \mathrm{H}_{10}$ & $13.3^{g}$ & 一 & 1.02 & 1.14 \\
\hline Anthracene & $\mathrm{C}_{11} \mathrm{H}_{10}$ & $14.4^{n}$ & $\approx 2$ & 0.67 & 0.78 \\
\hline Phenanthrene & $\mathrm{C}_{14} \mathrm{H}_{10}$ & $12.1^{i}$ & - & 0.68 & 0.74 \\
\hline Pyrene & $\mathrm{C}_{16} \mathrm{H}_{10}$ & $9.8^{\prime}$ & $\approx 0$ & 0.90 & 1.00 \\
\hline Fluoranthene & $\mathrm{C}_{16} \mathrm{H}_{10}$ & 11.7 & $\approx 7$ & 0.93 & 1.03 \\
\hline Triphenylene & $\mathrm{C}_{18} \mathrm{H}_{12}$ & 12.6 & $\approx 8$ & 0.98 & 1.12 \\
\hline Chrysene & $\mathrm{C}_{18} \mathrm{H}_{12}$ & $(14.9)^{k}$ & - & 0.69 & 0.77 \\
\hline Perylene & $\mathrm{C}_{20} \mathrm{H}_{12}$ & 13.8 & $\approx 5$ & - & - \\
\hline
\end{tabular}

a Values in parentheses have been evaluated by cryoscopic measurements.

${ }^{b}$ Observed from $C_{P}$ against $T$ plots.

- $V(c)$ is the observed molecular volume of the crystal at $T_{\mathrm{m}} ; V_{\mathrm{min}}$ is that required for molecular rotation on the basis of the smallest envelope of rotation; see references $14,25,26$, and 27 . a $V(1)$ is the molecular volume of the melt.

e Reference 21.

$t$ Reference 22.

$g$ Reference 29.

$n$ Reference 30.

$i$ Reference 31.

${ }^{3} \Delta S_{\mathrm{t}}=0.6 \mathrm{cal} \mathrm{mol}^{-1} \mathrm{~K}^{-1}$ for transition at $116 \mathrm{~K}$.

to Reference 32.

entropy of fusion would thus have a vibrational origin. Hence, spectroscopic study of solid and melt near the melting temperature are obvious desiderata.

Since reorientation is facilitated by a high degree of isotropy in the plane of the molecule, the nearly circular symmetry of coronene, for example, would be conducive to rotation of the molecule. Coronene does indeed have a thermal anomaly between 215 and $250 \mathrm{~K}$ which appears to be a motional transition in n.m.r. measurements whereas triphenylene and perylene are rigidly positioned in the lattice. ${ }^{(30)}$

Attempts to correlate melting parameters of these condensed polynuclear hydrocarbons with their molecular structures, molecular symmetries, etc., were disappointing. The entropy of melting of benzene $\left(8.5 \mathrm{cal} \mathrm{mol}^{-1} \mathrm{~K}^{-1}\right)$ is comparable to that of less-symmetrical, single-ring compounds such as thiazole, $\Delta S_{\mathrm{m}}=9.6 \mathrm{cal} \mathrm{mol}^{-1}$ $\mathrm{K}^{-1}$;(31) pyridine, $\Delta S_{\mathrm{m}}=8.5 \mathrm{cal} \mathrm{mol}^{-1} \mathrm{~K}^{-1}$;(32) furane, $\Delta S_{\mathrm{m}}=4.8$ and $\Delta S_{\mathrm{t}}=$ $3.7 \mathrm{cal} \mathrm{mol}^{-1} \mathrm{~K}^{-1}$; (33) thiophene, $\Delta S_{\mathrm{m}}=5.1$ and $\Delta S_{\mathrm{t}}=4.9 \mathrm{cal} \mathrm{mol}^{-1} \mathrm{~K}^{-1}$; (34) aniline, $\Delta S_{\mathrm{m}}=9.4 \mathrm{cal} \mathrm{mol}^{-1} \mathrm{~K}^{-1} ;{ }^{(35)}$ and toluene, $\Delta S_{\mathrm{m}}=9.2 \mathrm{cal} \mathrm{mol}^{-1} \mathrm{~K}^{-1}$,(36) where $\Delta S_{\mathrm{t}}$ is the entropy of transition. Likewise, the value for naphthalene $\Delta S_{\mathrm{m}}=$ $12.8 \mathrm{cal} \mathrm{mol}^{-1} \mathrm{~K}^{-1}$ is comparable with that of benzothiazole $\left(11.1 \mathrm{cal} \mathrm{mol}^{-1} \mathrm{~K}^{-1}\right)^{(37)}$ and of benzothiophene $\left(\Delta S_{\mathrm{m}}=9.2 \text { and } \Delta S_{\mathrm{t}}=2.4 \mathrm{cal} \mathrm{mol}^{-1} \mathrm{~K}^{-1}\right)^{(38)}$ under the same conditions. Due caution should be applied to values not based on direct calorimetric determination. However, as may be noted from the third column of table 6 , the molar entropy of fusion divided by the number of carbon atoms per molecule decreases rapidly at first and then more gradually with increasing number of carbon atoms and may reach an asymptote at 0.5 to $0.6 \mathrm{cal} \mathrm{mol}^{-1} \mathrm{~K}^{-1}$ ). 
Although harder to evaluate experimentally because of premelting effects, $\Delta C_{p, \mathrm{~m}}=\left\{C_{p}(1)-C_{p}(\mathrm{c})\right\}$ for the melting process tends to approach zero for the two substances with unusually small values of $\Delta S_{\mathrm{m}}$. Likewise, the experimental volume increments on melting $\Delta V_{\mathrm{m}}$ are also relatively small for these two substances.

A further not unrelated and tantalizing question concerns the molecular freedom of these planar molecules in the crystalline phase. Nuclear magnetic resonance ${ }^{(39)}$ and low-frequency Raman spectral data ${ }^{(40)}$ indicate that although benzene begins to reorient rotationally about its hexad axis just below fusion, naphthalene and anthracene do not do so about any axis. Raman spectra confirm, moreover, that torsional oscillations about the axis perpendicular to the plane are the least inhibited for benzene. Yet benzothiophene has been interpreted as doing so about all three axes. ${ }^{(41)}$ Of the three molecules studied in this paper, only pyrene shows a small thermal anomaly of unexplained origin accompanied by a slight change in lattice structure at $120.8 \mathrm{~K}$. The n.m.r. behavior over the range 255 to $285 \mathrm{~K}$ has been attributed by Fyfe et al. ${ }^{(42)}$ to the onset of reorientation of pyrene molecules about the axis perpendicular to the molecular plane with an activation energy of about $13.7 \mathrm{kcal} \mathrm{mol}^{-1}$. Camerman and $\operatorname{Trotter}^{(43)}$ found approximately $4^{\circ}$ angular amplitudes of oscillation about the long axis and about that perpendicular to the molecular plane but no evidence of statistical disorder. However, the presence of a disordered structure above $400 \mathrm{~K}$ has been reported. ${ }^{(44)}$ The above thermal data and other observations are considered evidence for the assertion that both the benzene and pyrene molecules, in contrast to other substances in table 6 , are rotationally reorienting in the crystalline phase.

These conclusions differ somewhat from those of Ubbelohde et al. ${ }^{(11,22-24)}$ who consider that only acenaphthene and triphenylene may have sufficient volume to reorient in the crystal and that in addition to these, benzene, naphthalene, pyrene, and fluoranthene, have room for "free rotation" only in the melt. Phenanthrene and chrysene, in principle, do not have room for such rotation even in the melt. In these two instances at least, vibrational entropy changes on melting may make substantial contributions to the entropies of melting. Phenanthrene ${ }^{(45)}$ also has recently been reported to show a small thermal anomaly of as yet unexplained origin near $342 \mathrm{~K}$. As previously noted, thermal data on the melting of coronene would also be of special interest.

The authors appreciate the partial financial support provided by the Division of Research of the United States Atomic Energy Commission.

\section{REFERENCES}

1. Inokuchi, H. J. Chem. Soc. Japan, 1951, 72, 552.

2. Inokuchi, H. Bull. Chem. Soc. Japan 1951, 24, 222.

3. Inokuchi, H.; Nakagaki, M. Bull. Chem. Soc. Japan 1959, 32, 65.

4. Jacobs, C. J.; Parks, G. S. J. Amer. Chem. Soc. 1934, 56, 1513.

5. Westrum, E. F., Jr.; Furukawa, G. T.; McCullough, J. P. In Experimental Thermodynamics, p. 133. J. P. McCullough \& D. W. Scott; editors. Butterworths: London. 1968.

6. West, E. D.; Westrum, E. F., Jr. In Experimental Thermodynamics, p. 333. J. P. McCullough \& D. W. Scott; editors. Butterworths: London. 1968. 
7. Westrum, E. F., Jr. J. Chem. Educ. 1962, 39, 443.

8. Robertson, J. M.; White, J. G. J. Chem. Soc. 1947, 358.

9. Chakravarti, S. C. Acta Crystallogr. 1954, 7, 378.

10. Klug, A. Acta Crystallogr. 1950, 3, 165.

11. McLaughlin, E.; Ubbelohde, A. R. Trans. Faraday Soc. 1957, 53, 628.

12. Fieser, L. F.; Novello, F. C. J. Amer. Chem. Soc. 1940, 62, 1855.

13. Kravchenko, V. Acta Physicochim. USSR 1947, 22, 187.

14. Mastrangelo, S. V. R.; Dornte, R. W. J. Amer. Chem. Soc. 1955, 77, 620.

15. Grosse, A. V.; Mavity, J. M.; Mattox, W. J. Ind. Eng. Chem. 1946, 38, 1041.

16. Bachmann, W. E.; Clarke, H. T. J. Amer. Chem. Soc. 1927, 49, 2089.

17. Pople, J. A.; Karasz, F. E. J. Phys. Chem. Solids 1961, 18, 28; 1961, $20,294$.

18. Oliver, G. D.; Eaton, M.; Huffman, H. M. J. Amer. Chem. Soc. 1948, 70, 1502.

19. Parks, G. S.; Huffman, H. M. Ind. Eng. Chem. 1931, 23, 1138.

20. Huffman, H. M.; Parks, G. S.; Barmore, M. J. Amer. Chem. Soc. 1931, 53, 3876.

21. Wong, W.-K.; Westrum, E. F., Jr. J. Phys. Chem. in the press.

22. Al-Mahdi, A. A. K.; Ubbelohde, A. R. Proc. Roy. Soc. Ser. A 1953, 220, 143.

23. Andrews, J. N.; Ubbelohde, A. R. Proc. Roy. Soc. Ser. A 1955, 228, 435.

24. Ubbelohde, A. R. Melting and Crystal Structure. Clarendon Press: London. 1965.

25. Frank, F. C. Trans. Faraday Soc. 1956, 52, 884.

26. Skau, E. L. J. Phys. Chem. 1935, 39, 764.

27. Goursot, P.; Girdhar, H. L.; Westrum, E. F., Jr. J. Phys. Chem. 1970, 74, 2538.

28. Parks, G. S.; Huffman, H. M. J. Ind. Eng. Chem. 1931, 23, 1138.

29. Washburn, E. W. (ed.). International Critical Tables, Vol. 4. McGraw-Hill: New York, 1928. p. 180.

30. Fyfe, C. A. Personal communication, June 1970.

31. Goursot, P.; Westrum, E. F., Jr. J. Chem. Eng. Data 1968, 13, 471.

32. McCullough, J. P.; Douslin, D. R.; Messerly, J. F.; Hossenlopp, I. A.; Kincheloe, T. C.; Waddington, G. J. Amer. Chem. Soc. 1957, 79, 4289.

33. Guthrie, G. B., Jr.; Scott, D. W.; Hubbard, W. N.; Katz, C.; McCullough, J. P.; Gross, M. C.; Williamson, K. D.; Waddington, G. J. Amer. Chem. Soc. 1952, 74, 4662.

34. Waddington, G.; Knowlton, J. W.; Scott, D. W.; Oliver, G. D.; Todd, S. S.; Hubbard, W. N.; Smith, J. C.; Huffman, H. M. J. Amer. Chem. Soc. 1949, 71, 797.

35. Hatton, W. E.; Hildenbrand, D. L.; Sinke, G. C.; Stull, D. R. J. Chem. Eng. Data 1962, 7, 229.

36. Scott, D. W.; Guthrie, G. B.; Messerly, J. F.; Todd, S. S.; Berg, W. T.; Hossenlopp, I. A.; McCullough, J. P. J. Phys. Chem. 1962, 66, 911.

37. Goursot, P.; Westrum, E. F., Jr. J. Chem. Eng. Data 1969, 14, 1.

38. Finke, H. L.; Gross, M. E.; Messerly, J. F.; Waddington, G. J. Amer. Chem. Soc. 1954, 76, 854.

39. Andrew, E. R. J. Chem. Phys. 1950, 18, 607; Andrew, E. R.; Eades, R. G. Proc. Roy. Soc. Ser. A. $1953,218,537$.

40. Fruhling, A. Ann. Phys. (Paris) 1951, 6, 401.

41. Guthrie, G. B.; McCullough, J. P. J. Phys. Chem. Solids 1961, 18, 53.

42. Fyfe, C. A.; Gilson, D. F. R.; Thompson, K. H. Chem. Phys. Lett. 1970, 5, 215.

43. Camerman, A.; Trotter, J. Acta Crystallogr. 1965, 18, 636.

44. Dutt, N. N. J. Chem. Phys. 1953, 21, 380.

45. Andrews, P. A.; Armington, A. F.; Rubin, B. Appl. Phys. Lett. 1965, 7, 86. See also Matsumoto, S.; Fukuda, T. Bull. Chem. Soc. Japan, 1967, 40, 743. 